\title{
Tuberculosis en trabajadores de la salud: importancia de los programas de vigilancia y control
}

\author{
Luis 0 strosky-Zeichner, M.D., ${ }^{(1)}$ M. Sigfrido Rangel-Frausto, M.D., M.Sc., (1) Elizabeth García-Romero, M.D., (1)
} Alma Vázquez, M.D., (1) M. Juana Ibarra, Lic. en Enf., (1) Samuel Ponce de León-Rosales, M.D., M.Sc. (1)

\section{Ostrosky-Zeichner L, Rangel-Frausto MS, García-Romero E, Vázquez A, Ibarra MJ, Ponce de León-Rosales S. Tuberculosis en trabajadores de la salud: importancia de los programas de vigilancia y control. Salud Publica Mex 2000;42:48-52.}

\section{Resumen}

Objetivo. D escribir los resultados de la vigilancia de tuberculosis en trabajadores de la salud en un centro hospitalario de tercer nivel. Material y métodos Se revisaron los registros de vigilancia de trabajadores durante 1992-1998, analizando variables demográficas, laborales, antecedentes clínicos, así como pruebas previas, prueba de la tuberculina (PPD), refuerzos y seguimiento. Como medida de asociación se utilizó la razón de momios (RM) con su respectiva significancia y los intervalos de confianza; la comparación entre diferentes subgrupos se realizó con la prueba $\chi^{2}$, y se determinó tiempo de conversión con análisis de Kaplan Meier. Resultados Se vigilaron 1617 trabajadores, 68\%, mujeres y $32 \%$, hombres. La edad promedio fue $26.9 \pm 7.6$ (15-68) años. Del total, $30.5 \%$ eran enfermeras; $14.6 \%$, médicos residentes, y $14.1 \%$, internos. Un $65.8 \%$ provenía del Distrito Federal. El 71.6\% tenía BCG y 15.1\%, PPD previo. El PPD al ingreso fue positivo en $39.6 \%$ de los casos; negativo, en el $48.3 \%$, y sin lectura, en el $12.1 \%$ restante. Se aplicaron 483 refuerzos (booster) en aquellos inicialmente negativos, y se encontraron 49 positivos. Se vigiló la conversión a PPD + por aplicaciones periódicas en 231 trabajadores, y se encontró dicha conversión en 100 de ellos (43.3\%). El tiempo promedio para detectar la conversión fue de $22.8 \pm 12.4$ meses. La tasa de conversión a los 12 meses fue de $20 \%$. Ú nicamente 50 trabajadores (50\%) recibieron y aceptaron profilaxis con isoniazida. Conclusiones. Un alto porcen-

\author{
Ostrosky-Zeichner L, Rangel-Frausto MS, \\ García-Romero E, Vázquez A, Ibarra MJ, \\ Ponce de León-Rosales S. \\ Tuberculosis in healthcare workers: \\ Importance of surveillance \\ and control programs. \\ Salud Publica Mex 2000;42:48-52.
}

\begin{abstract}
A bstract
Objective. To describe tuberculosis surveillance results among healthcare workers of a tertiary care center. Material and methods All medical records of workers from 1992-1998 were reviewed. D emographics, labor, medical history, previous testing, PPD, booster shots and follow-up were analyzed. Statistical analysis was performed with odds ratios, $p$-values, and $95 \%$ confidence intervals. Subgro up analysis were done with $\chi^{2}$. Kaplan-Meier estimates were used to analyze times to conversion. Results. Surveillance was done in 1617 workers (68\% female and 32\% male). Mean age was $26.9 \pm 7.6(15-68)$ years. Job positions were $30.5 \%$ nurses, $14.6 \%$ residents and $14.1 \%$ interns. Place of origin was Mexico City in $65.8 \%$. BC G vaccination was present in $71.6 \%$ and $15.1 \%$ had previous PPD.Admission PPD was positive in $39.6 \%$, negative in $48.3 \%$ and $12.1 \%$ were lost to follow-up. 0 n negatives, 483 booster shots were applied, and 49 additional positives were found. Follow-up was done in 231 workers, of which 100 (43.3\%) converted. The mean time for conversion was $22.8 \pm 12.4$ months. The conversion rate at twelve months was 20\%. Fifty workers received/ accepted isoniazid prophylaxis. Conclusions $A$ high percentage of workers were PPD-positive; booster shots allowed the detection of an additional 10\%.A high conversion rate underscores the need to organize tuberculosis control programs in Mexico.
\end{abstract}

(1) División de Epidemiología Hospitalaria y Control de Calidad de la Atención Médica, Instituto N acional de la N utrición Salvador Zubirán, México.

Fecha de recibido: 26 de mayo de 1999 - Fecha de aprobado: 8 de noviembre de 1999

Solicitud de sobretiros: Dr. Samuel Ponce de León, División de Epidemiología Hospitalaria y Control de Calidad de la Atención Médica, Instituto N acional de la N utrición Salvador Zubirán. Vasco de Quiroga 15,Tlalpan, 14000 México D.F., México. Correo electrónico: sponce@ quetzal.innsz.mx 
taje de trabajadores son PPD + al ingreso. Los refuerzos detectan $10 \%$ más de casos. La tasa de conversión enfatiza la necesidad de organizar estos programas en México.

Palabras clave: tuberculosis/vigilancia epidemiológica; tuberculina; servicios de salud; trabajadores; México
Kew words: tuberculosis/epidemiologic surveillance; tuberculin; health services; workers; M exico
L a tuberculosis pulmonar es un problema de salud pública remergente que ha tenido un gran impacto mundial. El resurgimiento de la enfermedad, su interacción con otros padecimientos, como el SIDA y la aparición de cepas multirresistentes ha generado un gran interés entre la comunidad científica por realizar estudios sobre sus formas de transmisión y métodos de prevención.

Uno de los grupos más vulnerables para adquirir la enfermedad es el de los trabajadores de la salud. El riesgo ocupacional de dichos trabajadores está determinado directamente por la exposición a pacientes infectados. ${ }^{1}$ Esta situación es especialmente alarmante por el reporte de brotes de tuberculosis adquirida en el hospital, tanto entre trabajadores de la salud como en pacientes. ${ }^{2}$ En Estados Unidos de América (EUA), en 1993, un estudio informaba que los trabajadores de la salud habían correspondido a 3.2\% del total de casos de tuberculosis. ${ }^{3}$ De hecho, actualmente existen programas obligatorios de vigilancia de la tuberculosis en la mayoría de los hospitales de ese país. ${ }^{4}$ La vigilancia debe incluir una valoración inicial al ingreso y seguimiento periódico. El método más reconocido para efectuar esta vigilancia es el de la aplicación periódica de tuberculina (derivado proteico purificado, PPD, por sus siglas en inglés) y la investigación exhaustiva con rayos $\mathrm{X}$ de tórax, así como las baciloscopías y los cultivos en los casos indicados. La aplicación de refuerzos (booster) en sujetos con PPD negativo ha demostrado ser una herramienta imprescindible para detectar falsos negativos en la valoración inicial. ${ }^{5,6}$ Hay relativamente poca información sobre la positividad a PPD basal y las tasas de conversión aceptables para un hospital. ${ }^{7}$ Sin embargo, hospitales grandes han notificado alrededor de $40 \%$ de positividad inicial con menos de $5 \%$ de conversión anual. ${ }^{8-10}$

En México se cuenta con muy poca experiencia al respecto. En 1994, nuestro equipo de trabajo hizo una valoración inicial de 200 trabajadores de la salud, en la que se encontró una positividad a PPD basal de $70 \%$ y se identificaron como factores de riesgo una edad mayor de 30 años y la historia de vacunación con bacilo de Calmette Guerin (BCG) ${ }^{11}$ El objetivo de este trabajo es mostrar los resultados del programa de vigilancia de tuberculosis nosocomial en trabajadores de la salud que se lleva a cabo en el Instituto Nacional de Nutrición Salvador Zubirán (INNSZ), que actualmente mantiene un número considerable de trabajadores bajo vigilancia, lo que permite tener una dimensión longitudinal debido a que se cuenta con seguimiento de algunos de estos trabajadores hasta por seis años.

\section{Material y métodos}

El INNSZ es un centro hospitalario de tercer nivel con 160 camas censables. En él se tratan casos médicos y quirúrgicos de alta complejidad, constituye un centro de referencia nacional, y se atienden en promedio 30 casos de tuberculosis pulmonar por año. Aunque deben de ingresar a cuartos de aislamiento especiales con presión negativa, los pacientes con tuberculosis frecuentemente pasan algún tiempo en el servicio de urgencias o en otras áreas de hospitalización hasta que se detecta la enfermedad o haya disponibilidad de cuartos especiales. El INNSZ cuenta con aproximadamente 2000 trabajadores, entre los que se incluye el personal médico y de enfermería, el personal de intendencia, el administrativo y de servicios, así como estudiantes. El hospital cuenta con una división de epidemiología hospitalaria y control de calidad de la atención médica, que vigila infecciones de adquisición nosocomial tanto en pacientes como en el personal. Desde 1992, se mantiene un programa de vigilancia de tuberculosis en el personal, que incluye la aplicación de un cuestionario sobre antecedentes sociodemográficos, la revisión de la cicatriz de BCG, la identificación de factores de riesgo y preguntas sobre la exposición previa a tuberculosis, así como la aplicación rutinaria de PPD al momento del ingreso al INNSZ. Por otro lado, se lleva un seguimiento anual en personas que tuvieron PPD negativo al ingreso y se registra el resultado anual hasta que se convierten en PPD positivas. En caso de conversión se ofrece rutinariamente profilaxis con isoniazida. Asimismo, se explora la reacción de refuerzo en personas con PPD negativo al in- 
greso, con una segunda aplicación de PPD en las dos semanas siguientes a la primera en que se aplicó el tratamiento. La información se registra en libretas y en una base de datos diseñada para el seguimiento.

Se revisaron los registros de todos los trabajadores en la base de datos, para describir el perfil sociodemográfico, los antecedentes clínicos, la prevalencia de PPD positivo al ingreso, la frecuencia de reacción de refuerzo positiva, la frecuencia de conversión a PPD positivo y la administración de profilaxis o tratamiento. El análisis descriptivo, el cálculo de la razón de momios (RM) y la comparación entre diferentes subgrupos se realizó con la prueba de $\chi^{2}$. Además, se efectuó análisis de Kaplan-Meier para determinar el tiempo de conversión.

\section{Resultados}

De 1992 a 1998 se vigilaron 1617 trabajadores. La edad promedio fue de 26.96 \pm 7.6 (15-68) años, y el resto de las principales características sociodemográficas se muestran en el cuadro I. De la población estudiada, $71.6 \%$ contaba con historia de aplicación de vacuna $B C G$, aunque sólo $62.1 \%$, mostraba cicatriz de ésta. El 15.1\% notificó que le habían realizado la prueba de PPD en otro centro hospitalario, en algún momento previo al ingreso al trabajo en el Instituto. Diez trabajadores mencionaron haber padecido tuberculosis pulmonar confirmada y tratada antes de su ingreso. Del total, $50 \%$ admitieron tener un COMBE positivo. Los resultados de la aplicación inicial de PPD, los refuerzos y el seguimiento anual se muestran en la figura 1. Las variables sociodemográficas que se relacionaron con PPD positivo basal fueron edad $>30$ años (RM 2.97; IC95\% 2.30-3.82, $p=0.000001$ ), vacunación con BCG (RM 1.67; IC95\% 1.23-2.26, $p=0.0005$ ), categoría enfermera (RM 2.0; IC95\% 1.41-2.83, $p=0.00004$ ) y COMBE (RM 1.5; IC95\% 1.19-1.89, $p=0.0004$ ). Los trabajadores con PPD positivo tuvieron una induración promedio de $19.74 \mathrm{~mm} \pm 8.07 \mathrm{y}$ en los negativos la induración promedio fue de $1.37 \mathrm{~mm} \pm 2.95$ (rango de 0-9.5). De los 231 trabajadores que tuvieron seguimiento anual, 100 (43.3\%) convirtieron a PPD positivo. El tiempo promedio para detectar la conversión en estos trabajadores fue de 22.8 \pm 12.4 (rango de 2-60) meses. El tiempo de conversión se muestra en la figura 2. A los 12 meses, $20 \%$ de los trabajadores convirtieron. No hubo variables sociodemográficas o laborales que se asociaran a riesgo de conversión. Sólo 50 trabajadores recibieron o aceptaron recibir profilaxis con isoniazida (cuadro II). En dos trabajadores se diagnosticó tuberculosis pulmonar confirmada por

\section{Cuadro I \\ Características sociodemográficas \\ DE LOS TRABAJADORES EN VIGILANCIA EPIDEMIOLÓGICA \\ PARA tUberculosis. InStituto Nacional de La \\ Nutrición Salvador Zubirán, México, 1992-1998}

No. $\%$

$\begin{array}{ll}\text { Población total } & 1617\end{array}$

Sexo

\begin{tabular}{lrr} 
Hombres & 517 & 32 \\
\hline Mujeres & 1100 & 68
\end{tabular}

Categoría

$\begin{array}{lll}\text { Enfermeras } & 493 \quad 30.5\end{array}$

Médicos residentes $\quad 237 \quad 14.6$

Médicos internos 22814.1

Afanadoras $133 \quad 8.2$

$\begin{array}{llll}\text { Estudiantes de enfermería } & 103 & 6.4\end{array}$

$\begin{array}{lll}0 \text { tros } & 423 & 26.2\end{array}$

Procedencia

Distrito Federal $\quad 1066 \quad 65.8$

\begin{tabular}{lrr} 
Estado de México & 65 & 4.0 \\
\hline Jalisco & 55 & 3.4 \\
\hline Veracruz & 42 & 2.6 \\
\hline O tros estados & 150 & 9.4 \\
\hline Extranjeros & 19 & 1.2 \\
\hline N o disponible & 220 & 13.6
\end{tabular}

No disponible

22013

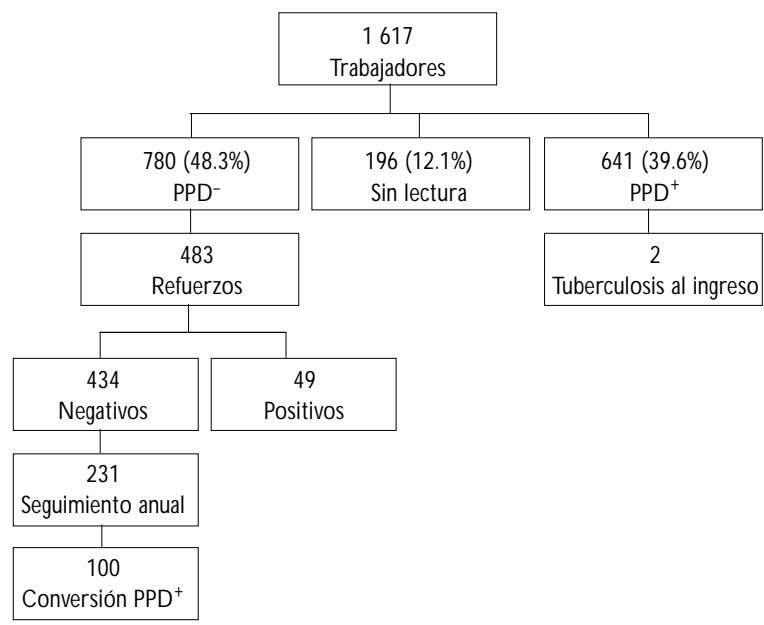

Figura 1. Resultados del programa de vigilancia EPIDEMIOLÓgICA DE TUBERCULOSIS. INSTITUTO NaCIONAL de la Nutrición Salvador Zubirán, México, 19921998 


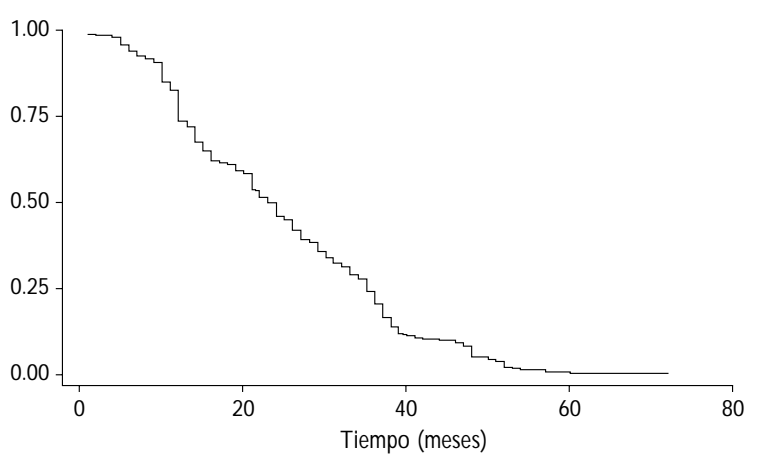

Figura 2. Tiempo de conversión a PPD positivo en tRABAJADORES DE SALUD. INSTITUTO NACIONAL DE LA Nutrición Salvador Zubirán, México, 1992-1998

\section{Cuadro II \\ Trabajadores que convirtieron a PPD positivo. Instituto Nacional de la Nutrición Salvador Zubirán, México, 1992-1998}

$\begin{array}{ccc}\text { Iniciaron } & \text { No } & \text { Inicio tratamiento } \\ \text { profi- } & \text { iniciaron } & \text { con antifímicos } \\ \text { laxis } & \text { profilaxis } & \text { por TB pleural } \\ \text { (30) } & \text { (22) } & \text { (1) }\end{array}$

\begin{tabular}{lccc} 
Enfermeras & 6 & 11 & \\
\hline Estudiantes de enfermería & 3 & - & 1 \\
\hline Médicos residentes & 11 & 4 & \\
\hline Administración & 2 & - & \\
\hline Médicos adscritos & - & 1 & \\
\hline Afanadores & 4 & 2 & \\
\hline Internos & 4 & 3 & \\
\hline Químicos & - & 1
\end{tabular}

radiografías y cultivos al ingreso, y ambos recibieron tratamiento completo con curación.

\section{Discusión}

Este estudio muestra que la vigilancia epidemiológica de tuberculosis en trabajadores de la salud es una actividad relevante. Encontramos una positividad basal de PPD cercana a 40\%. Esta cifra es sorprendentemente similar a la de hospitales en EUA y notablemente más baja a la informada previamente en nuestro hospital. Esto probablemente se explica por la importante ampliación de la muestra (200 vs. 1 617) y por la similitud de nuestro hospital en cuanto a características de atención por nivel de complejidad con los hospitales mencionados. Un dato interesante, que se notifica con poca frecuencia, es que $12.1 \%$ de los trabajadores no acudieron a la lectura de la prueba. Esto refleja claramente las dificultades que estos programas enfrentan para el seguimiento de los sujetos. En la valoración inicial se detectaron dos casos de tuberculosis activa. Esto significa que para detectar un caso se tuvieron que aplicar poco más de 800 pruebas. Más que analizar el costo-beneficio de esta intervención, se debe pensar en que estas dos personas recibieron tratamiento y se evitó la transmisión nosocomial tanto a pacientes como a compañeros de trabajo, lo que justifica ampliamente este tipo de evaluaciones.

Las variables que estuvieron asociadas a positividad basal de PPD fueron la categoría de enfermera (probablemente en relación con exposición laboral previa), la historia de COMBE y la vacunación previa con BCG. Sin embargo, esta última no ha sido caracterizada como factor de interferencia para la interpretación de la prueba con PPD. ${ }^{12}$ Resulta interesante haber comprobado la utilidad del antecedente de COMBE como factor de riesgo para exposición a la enfermedad.

La aplicación de refuerzos a los sujetos con PPD negativo ocurrió en poco más de la mitad de éstos, que permitió identificar a 49 sujetos $(10 \%)$ con positividad basal (falsos negativos), que de otra forma habrían sido considerados posteriormente como convertidores, tal vez con profilaxis innecesaria. En forma similar, otros estudios han notificado la detección de hasta $10 \%$ más de positivos. ${ }^{6}$ La aplicación de refuerzos debe ser incorporada a los programas actuales de vigilancia.

La cifra de conversión a PPD positivo (43.3\%) en los sujetos negativos con seguimiento anual en el total de años de vigilancia, es alarmante. Esto significa que la mitad de los trabajadores previamente no infectados se contagian en los primeros dos años de trabajo dentro del hospital. Esta cifra es mucho mayor a lo notificado en otros centros. ${ }^{13,14}$ Esto probablemente significa que nuestra población de pacientes es de alto riesgo y las medidas de protección han sido insuficientes. Llama la atención que sólo 50 trabajadores aceptaron recibir isoniazida, lo que nos indica que tal vez hay que hacer un mayor énfasis en los criterios de administración y educación sobre la importancia de la profilaxis.

Otros estudios en nuestro país hechos en hospitales de Guadalajara y Monterrey ${ }^{15,16}$ han documentado frecuencias similares a las nuestras, lo que demuestra la relevancia de este problema en el ámbito nacional.

Este trabajo aporta información relevante sobre la vigilancia de tuberculosis entre trabajadores de la salud en México y muestra la utilidad de los progra- 
mas de este tipo. Idealmente todos los hospitales del país deberían llevar a cabo estos programas y la información debería de ser concentrada para producir informes representativos y de relevancia nacional e internacional.

\section{Referencias}

1. Sepkowitz KA. Tuberculosis and the health care worker: A historical perspective. Ann Intern Med 1994;120:71-79.

2. Centers for Disease Control and Prevention. Nosocomial transmission of multidrug-resistant tuberculosis among HIV-infected personsFlorida and N ew York, 1988-1991. MMW R Morb Mortal W kly Rep 1991;40:585-591.

3. Centers for Disease Control and Prevention. Expanded tuberculosis surveillance and tuberculosis morbidity- United States, 1993. MMW R Morb Mortal W kly Rep 1994;43:361-366.

4. Centers for D isease Control and Prevention. G uidelines for preventing the nosocomial transmission of Myco bacterium tuberculosis in healthcare facilities, 1994. MMW R Morb Mortal W kly Rep 1994;43(RR-13): 1-132.

5.Thompson N J, G lassroth JL, Snider DE, Farer LS.The booster phenomenon in serial tuberculin testing. Am Rev Respir D is 1979; 119:587-597. 6. Sepkowitz KA, Feldman J, Lo uther J, Rivera P,Villa N , D eH ovitz J. Beneffit of two-step PPD testing of new employees at a N ew York City hospital. Am J Infect Control 1997;25:283-286.

7. Lowenthal $G$, Keys T. Tuberculosis surveillance in hospital employees: Are we doing too much? Infect Control Hop Epidemiol 1986;7:209-211.
8. Sepkowitz KA, Fella P, Rivera P, Villa N, D eH ovitz J. Prevalence of PPD positivity among new employees at a hospital in N ew York City. Infect Control Hosp Epidemiol 1995;16:344-347.

9. W urtz R, Fernandez J, Jovanovic B. Real and apparent tuberculin skin test conversion in a group of medical students. Infect Control Hosp Epidemiol 1994;15:516-519.

10. Louther J, Rivera P, Feldman J,Villa N , D eH ovitz J, Sepkowitz KA. Risk of tuberculin conversion according to occupation among health care workers at a N ew York City hospital. Am J Respir C rit Care Med 1997; 156: 201-205.

11. Mo lina-G amboa J, Rivera-Morales I, Ponce-de-Leon-Rosales S. Prevalence of tuberculin reactivity among health care workers from a Mexican hospital. Infect Crontrol Hosp Epidemiol 1994;15:319-320.

12. Centers for D isease Control and Prevention. Tuberculin skin test survey in a pediatric population with high BCG vaccination coverage-Botswana 1996. MMW R Morb Mortal W kly Rep 1997;46:846-851.

13. Ramirez JA, Anderson P, Herp S, Raff MJ. Increased rate of tuberculin skin test conversion among workers at a university hospital. Infect $C$ ontrol Hosp Epidemiol 1992;13:579-581.

14. Raad I, C usick J, Sherertz RJ, Sabbagh M, Howell N . Annual tuberculin skin testing of employees at a university hospital:A cost-benefit analysis. Infect Control Hosp Epidemiol 1989;10:465-469.

15. G arcía-Reza R, Magro M, Rivera-Morales I, Rendon-Perez A. Incidencia de conversión al PPD en residentes del Hospital Universitario (resumen). En: Memorias del XXII Congreso Anual de la Asociación Mexicana de Infectología y Microbiología Clínica. Enferm Infecc Microbiol Clin 1997; 17:107.

16. Esparza-A humada S, 0 liden-Ramírez L, Hernández-Moreno AL, Péerez-Gómez R, Rodríguez-N oriega E. Conversión de PPD en trabajadores de la salud en un hospital de alto riesgo aumentado para adquirir tubercuIosis (resumen). En: Memorias del XXI Congreso Anual de la Asociación Mexicana de Infectologia y Microbiologia Clínica. Enferm Infecc Microbiol Clin 1996;16:48. 